\title{
Mobile Application Accelerated by Cloud Based Systems
}

\author{
Hira Arshad \\ Fatima Jinnah Women University \\ The Mall Rawalpindi, Pakistan \\ Hira.arshad9@gmail.com
}

\author{
Muqaddas Naz \\ Fatima Jinnah Women University \\ The Mall Rawalpindi, Pakistan \\ muqadasawan532@yahoo.com
}

\author{
Madiha Arshad \\ Fatima Jinnah Women University \\ The Mall Rawalpindi, Pakistan \\ madiha.arshad7@gmail.com
}

\begin{abstract}
Networking process, phone sensor like GPS reading, these mobile applications is much expensive in references of energy and due to this, mobile phone not much able to provide better mobile services to the user due to this embedded sensor. In order to provide better mobile application high capacity for computation is required and developer of mobile find many difficulties in the development of mobile application. These application demands for high capacity also restrict the mobile developers. In the previous years, these problems has focused by different researcher by using the technique of cloud computing. Cloud computing is a way of computing different mobile application whereas the applications have delivered like a services over the hardware, internet and software. To increase the use of mobile computing it is difficult due to problems of mobility and disconnectivity. Mobile cloud computing (MCC) is a way that solves such problems by enhancing mobile application. MCC is a mixture of mobile computing, cloud computing \& wireless network that is use to bring resources to network operator, users of mobile \& providers of cloud computing.
\end{abstract}

Keywords: Cloud Testing, Cloud Media, mobile computing, cloud trust management system, cloud mobile media.

\section{INTRODUCTION}

In recent years there has been notable increase in the use of mobile application, mobile application use in many fields in order to provide different type of facilities. Mobile now-a-days use in every field in medical field, electronic banking, EMS (Emergency Managing System) and these all fields are related to human access. While providing different services via mobile there is also many problems that need to be address [4].Major critical condition when development process is complex and demands more cost, to address such kind of problem some technology must be used [11]. Human oriented facilities can provide effectively by the use of technology. Different type of technology has been surveyed in order to increase the usability of mobile device and make enhancement in mobile application. Cloud computing is the technology that played a significant role that helps to provide better mobile services to the users [1].

Cloud computing portent a latest period at where storage, exploitation, access, sharing of the information is in wider range and these all done in less cost. Cloud computing is cost effective, much user friendly and also secure. By using the cloud computing technique resources can be easy to allocate by virtual-zing [6]. Cloud computing also help to scale-up the recourses of the whole system. Cloud computing is cost effective and this is the main advantage of it. The advantage of cloud computing that it scales up/down the resources and it can also migrate virtualized servers without making the influence on users[7]. In cloud testing the resources are shared with the bandwidth, central memory.

The grouping of wireless network communication, convenient system device, infrastructure and cloud computing make the basis for model of the computer, this called mobile cloud computing. MCC grants many users to avail boundless power of computing [3]. By adding feature of the cloud computing in the mobile field, we explain it as: 
"Mobile cloud computing is a model for transparent elastic augmentation of mobile device capabilities via ubiquitous wireless access to cloud storage and computing resources, with contextaware dynamic adjusting of offloading in respect to change in operating conditions, while preserving available sensing and interactivity capabilities of mobile devices."

Information technology field is enhancing day-by-day, cloud computing and architecture that is service oriented are putting significant role in the development of IT. Many system services providers are now using the technology of cloud computing to install system services on internet for the users [5]. Cloud computing have provide best facility to the media services providers, cloud computing gives a better way out to decrease cost of starting payment $\&$ operate network of mobile media [6]. Cloud computing provides a way of randomly allocating the resources of the system in order to fulfill the flexible demand of application.

In medical field health care providers get advantage from the MCC in the classification to create vital decision in healthcare, to get patient data timely, access of the exact information about the patient based on the networking technology like cloud computing services [4]. MCC make it comfort to get the data of the patient instantly from anywhere by using any device. This paper apprehension on the technology of cloud computing and how it helps to enhance the mobile services in order to facilitate the users which belong to any field.

\section{Survey Papers Summaries}

\subsection{Cloud based Food Calorie Measurement [4]}

Mobile applications are use for patient in order to check their vital sign, illness, anticipation and also for treatment purpose.MCC use for providing the services of health by using mobile to defeat the issues of interoperability that occur in different format of information. System that measures the food calorie introduced that based on cloud computing of mobile. To recognize the calorie two things use, cloud vector mechanism (SVM) and technique of Map Reducing. SVM is a mechanism for training in cloud computing and Map Reduce use for the learning of machine. Cloud computing have advantage that it allows the user to admittance infrastructure and software such as storage, server $\&$ application program that given by providers of cloud at very less cost. MCC removes the obstacles from the mobile environment, performance and with the help of MCC user can communicate in different format of information. System that is based on cloud computing can grant the patients \& health professional to admittance the medical report.

\subsection{Detection of Sybil Attacks in Participatory Sensing Using Cloud based Trust Management System [5]}

Mobile device has sensing capability like microphone, GPS, camera, accelerometer etc. this kind of technologies helps the user to collect things. User who integrates client application on their mobile device can enter the data. Mobile device provides a very well environment to participatory sensor Apps. An excess exciting participatory sensor Apps used for health anticipation over few years. Balance \& sensing in health care are the two example of the participatory sensor Apps. These two are used to gather \& share the data of health. Due to participatory sensor anyone can contribute \& explore the mobile Apps that cause malevolent attack. Harmful participators make mobile device position undesirable. At the time when sensor gathering the readings malicious participator add harmful data. Malicious participator that has different identities uses these identities to operate spitefully. Malicious participator steals information or gives the data which is incorrect that is Sybil Attack. So, any malicious participator sensor network can add wrong data over the network that puzzle network Apps and cause trust issues. To solve this trust issues CbTMS (Cloud-based Trust Management System) introduced in order to evaluate trust of network participator in participating sensor Apps. CbTMS have advantage gives elastic infrastructure, by using this elastic infrastructure the problem of malicious participating sensor network can easily eliminate.

\subsection{An Android-Enabled Mobile Framework for Ubiquitous Access to Cloud Emergency Medical Services [3]}

'Emergency Medical System' (EMS) perform activities that are related to the patients. EMS have the most vital process, activities from making an emergency call for the services of an ambulance to the discharge of the patient from the hospital and these are almost interconnected this is the reason that management is much critical for both the emergency services provider and for the patient. For this 
purpose cloud computing services perform a significant role and also the mobile based technology. MCC solve such vital issues that occur while providing the emergency services to the patient. EMS will be available by mobile android based device and gives the notification to the care provider whenever the vital data about the patient come this is a feature that proficient the resources of the mobile device and this feature achieve by MCC message mechanism. This mechanism plays an important role it communicate the server to the application of the mobile device that runs on Android. EMS system makes attention toward the MCC in order to increase the elasticity of their emergency system. It has advantage of offering ease in the access of record of the health management with low cost.

\subsection{Cloud Mobile Media: Reflections and Outlook [6]}

Mobile device provides the video content, people understand that the mobile videos are controlled by three challenges: strong media don't fitted due to limited mobile resources. Second, bandwidth of communication among mobile limited due to the untrustworthy channels. Third, popular content not achieve due to the static mechanisms of resources of the system. To enhance the network of mobile media new technology and ideas are required in order to facilitate the user with more good quality, end users claim more enhanced services and more better quality cloud computing plays a significant role in order to provide more enhancement in the field of media. The cost of the network of mobile media must be set aside low; the cost of starting payments of mobile media should be low if the cost is high then that cost will bear by the services provider that will influence the media access between the media users. MCC have provide best facility to the media services providers, gives a better way out to decrease cost of starting payment \& operate network of mobile media. MCC did this by randomly allocating the resources of the system in order to fulfill the flexible demand of application.

\subsection{SC4Cloud Tooling: An Integrated Environment for the Development of Business Processes With Security Requirements in the Cloud [7]}

Cloud computing and architecture that is service oriented are putting significant role in the development of IT. Architecture that is service oriented use different techniques in order to run the specification of business process. Whenever dealing with critical data security concern is very important, growing requirement in security providence need more enhanced technology and the best way to solve such security requirement cloud computing plays a significant role. To make continuous modification in models of business process and to provide security and provide better services these all are exclusive and time overriding. Applicable reason is this that different stakeholder involves in making and running of security business process and different stakeholder gives different requirements which is expensive. Major resource for computation required in order to provide business process along the security need and these requirement for security with less time can be fulfill by the technology of cloud computing.

\subsection{Mobile Cloud Computing: A Comparison of Application Models [2]}

Dominant application can run by the user that allowed by the mobile device that get benefit from the exchange of better data of mobile device. Mobile device application used many task e.g. edit, storage, transmitting, videos upload, health management etc. Mobile hardware, software \& network of mobile keep on improving, resources of mobile device always be poor, not much secure, connection is unbalanced \& also having less energy. Shortage of resources always is main problem in the mobile application, so mobile computation forever includes compromise. In order to remove such mobile application problem cloud computing plays a significant role. MCC act as a model that ensures the convenience to compute the resources. It provide the better services, process ability on the internet, and reduction of cost, storage capacity increases, system that is automatic, and more flexibility in services.

\subsection{Assessment of Today's Mobile Banking Applications from the View of Customer Requirements [9]}

Banking through mobile is division of the electronic banking that underlies commerce of mobile condition. User have many requirements and hope from the application of mobile. Many of the user requirements not much easily fulfilled by the mobile service provider, some technology required for it, and the best facilities provide by using the cloud computing. Improvement must be done in the banking application that mobile device provides, user need more flexibility, more fast transition in 
much less time and want these in low cost. Banking application integrated in the mobile device that provides user help to access bank services by using the mobile device. Application of mobile has to be particularly measure in order to fulfill needs of the users, and also put particularly restriction on mobile device application and on communication of mobile. For such kind of purpose MCC technology use in order to accommodate the users of banking application. MCC helps electronic banking finance services that implemented through the internet, with the time reduction and more ease for users.

\subsection{On Some Key Requirements of Mobile Application Software [8]}

MCC requires more in software in order to expand design and sustain services of mobile. Quality of services should be improved rather than making change in the location of the software of client. Techniques should be used to achieve properties for mobile application that are cost effective. key properties of cloud computing services is providing the correctness, similarity among the exact behavior and services request that could experiential in the system that is selected to provide the services. Another property that is relevant to the correctness is the stoutness which is distinct as the program attribute. Requirement for the system that is dependent is much apparent while in view of the increase in complexity in the requirement of the system. MCC use in order to provide more services and fulfill all requirements of the users without the complexity within less cost.

\subsection{Cloud based Mobile Application Testing [10]}

Mobile Apps that invented needs to be test because users expect more quality. To test the mobile Apps is difficult task for the developer, to test the mobile application by first keeping diversity of mobile device in mind and the resource which developer needs to test the mobile application is different from one mobile phone to another. In previous, simulators for mobile Apps only check the functionality of OS, simulators not simulate speed, size of cache, cores of mobile device. To address such problems cloud testing uses. Traditional testing is much different from cloud testing due to the severe change in mobile device hardware. Mobile Apps testing and normal testing are different because of the limitation of the hardware. Mobile Apps testing process is challenging to ensure that the application is error free is costly $\&$ also the time overriding. So, cloud computing testing use because cloud computing is cost effective. MCC test mobile application by using infrastructure. In the cloud testing the user has no need to be alert about the technology used and about the infrastructure. Testing is use for simulation of mobile application. In cloud testing the resources are shared with the bandwidth, central memory. The advantage of cloud computing that it scales up/down the resources and it can also migrate virtualized servers without making the influence on users.

\subsection{Mobile Cloud Computing [1]}

To increase the use of mobile computing it is difficult due to problems of mobility and disconnectivity. Mobile cloud computing (MCC) is a way that solves such problems by enhancing mobile application. MCC is a mixture of mobile computing, cloud computing \& wireless network that is use to bring resources to network operator, users of mobile \& providers of cloud computing. mobile application such as natural language, search for multimedia, social networking, sensor data, processing of images, computing of crowd, sharing of GPS, speech synthesis these all requires much high capacity for computation and due to this the developers of mobile application find difficulties in implementation of mobile application. These application demands for high capacity also restrict the mobile developers. Different techniques used to eliminate such issues. To address these issues proposed solution must be reviewed and should also explore new challenges that occur in cloud computing of mobile.

\subsection{Security of Cloud Computing, Storage, and Networking [11]}

Cloud computing has diversified applications in a field of mobile and computing applications. It provides a better and enhancement feature of multiple applications in a mobile and computer. Such type of technologies helps to get a better transmission and storage of the contents using a different ways. Due to diversified use and scope of cloud computing hence it is more liable to be attacked by suspicious source. It is a risk to a secure network and interface. Hence all the security terms e-g secure, protect, safe to use, reliable, authentication and independency are conflicting in aspect of cloud computing. Hence a different scenarios and techniques have been introduced in this paper to achieve security along cloud computing that will definitely be an ideal system having cloud computing with complete safety, privacy and protection. 


\subsection{Cloud-Based Augmentation for Mobile Devices: Motivation, Taxonomies, and Open Challenges[12]}

Mobile computing has many advantages as it has armed the world with the power and versatility. Many researches have been done yet to explore this field. Augmented mobile devices technology enables the far away communication, large computations and to store a large amount of data over the cloud. In this paper, there is a brief study on CMA such as mobile augmentation and taxonomy .The paper has been concluded as giving variety of ideas to enhance mobile cloud computing using CMA such as storage, processing and security. It also describes certain deficiencies tat how can we improve the interface and maintainability issues.

\subsection{Privacy Arguments: Analysing Selective Disclosure Requirements for Mobile Applications. [13]}

This paper reflects a very important factor of privacy in mobile applications. Sometime requirement elicitation process doesnot bother to keep in mind the most important factor that is of privacy because it's not easy to gather such requirements because of its dynamic range and versatile audience. In this paper, it is been tried to resolve this issue by introducing a privacy arguments that would take care of personal information disclosure regarding to contents and context. Research have been done in this paper by considering the privacy requirements of a Buddy tacker that is a sort of mobile application. Due to such privacy requirements, a user can share its personal information under terms of privacy preferences. This paper suggests the idea that a user can give well defined requirements even at runtime. Mobile devices can look up to web apps by relating software's to achieve privacy.

\subsection{Mobile Cloud Computing as Future for Mobile Applications [17]}

In the past few years mobile has been the most important part of the society as it has a wide range of user all over the globe so different factors must be keep in mind. One of the most important factor is continuously modifying the mobile embedded technologies and to facilitate the user with more comfortable and modified set of applications via mobile. An emerging technology has a wide demand for mobile applications that is Cloud computing that is very useful for mobile applications in a present era. Cloud computing is the most beneficial technique for the mobile cloud computing environment. This paper discusses the facts that how different issues such as performance, security and different challenges can be resolved and suggest different idead such as energy storage in mobile devices and migration issues.

\subsection{Software Trustworthy Testing based on Cloud Testing [20]}

Testing has always been an essential part in software development since centuries. As it reflects the great essence of the product being developed. Product testing leads to software quality assurance that will ensure the performance. Reliability, usability and maintainability of the software. It's been observed that software testing is very important factor to win out the user trust. Cloud testing is a testing technique used for testing a cloud computing systems that include the service applications over cloud and cloud. This paper will answer the certain questions that are most probably raised by many engineers, managers and tester and this paper also gives the objectives, benefits and requirements in cloud computing system. This paper also discusses that which software needs to be tested by cloud testing.

\subsection{Cloud Computing in Mobile Applications [19]}

There are two factors involved in the implementation of any technology that how it is going to be implemented and what effect would it give after its implementation. Cloud computing is a new emerging technology for mobile applications and we will consider these two factors for its implementation that how it is going to be implemented in mobile devices and after that how mobile devices will access the cloud for transmission and processing of applications. The major challenge that is going to be faced after its implementation is the security hazards because of its diversified range of applications.

\subsection{Research on Mobile Cloud Computing: Review, Trend and Perspectives [16]}

Mobile computing and the cloud computing has become the new emerging technology, also known as (MCC).This technology has many advantages such as processing of applications and storage over the cloud. Yet it involves many risks such as security threats. So a lot of research work has been done on 
this idea having past trends and for the sake of future research. It has been concluded that still most of the areas of this technology are unexplored.

This paper includes a research on this MCC and gathers information about the infrastructure and features of mobile computing. The paper has been concluded by giving some suggestions over the virtualization of images and the communication over wireless and wired networks.

\subsection{Using Activity Theory In Analyzing Requirements For Mobile Phone Application [18]}

The bottom line of this paper is to solve the issues that are created during requirement elicitation such as incomplete understanding of user's requirement for different mobile applications. For this purpose an activity theory is used to completely understand and analyze the user's requirement completely. Requirements after elicitation have to be chunked up in an activity diagram so this paper focuses on the importance of activity theory in requirement elicitation for mobile computing applications. Activity diagram can reflect non functional requirements along functional requirements and highlights each feature.

\subsection{Testing Requirements For Mobile Applications [15]}

Communication among the mobile devices is the wireless communication that enhances the level application developed for the mobile devices. With the enhancement of mobile applications due to wireless communication, there are also certain factor that needs to be entertained for this purpose such as battery, processing and storage. For this purpose a software requirement testing is required to enhance these features. The paper has been concluded by suggesting some specific techniques for requirement elicitation and then software testing.

\subsection{Usage Control Requirements in Mobile and Ubiquitous Computing Applications [14]}

This paper involves a study of usage control means that the conditions and constraints that to whom the content should be visible, it is usually referred when there is an involvement of third party. Usage control requirements means that the elicitated requirements for mobile cloud and ubiquitous computing must be classified on the basis of contents and the access specified for example you can put constraint on the data that this data is going to be used only for statistical purpose. For this purpose different scenarios and techniques has been introduces to carry out the usage control requirements.

\section{ANALYSiS}

Mobile cloud computing is the new emerging technology for mobile devices that enables to enhance certain characteristic of mobile devices. To completely understand and explore the mobile computing, we have reviewed 20 techniques and consider 11 parameters to evaluate it.

Table 2 reflects the evaluation criteria based on the parameters described in table 1.

Analysis discloses that how the security and privacy can be achieved with the mobile cloud computing as stated in Shih-Hao Chang et al[5], F. Lins1 et al[7], L.Murugesan et al[10], M. Hamdi et al[11],A.K. Bandara et al [13], M. Hilty et al[14], H.Q Abdullah et al [16],V. Lelli Dantas et al [15], D. Sahu et al [19], A. Mohsenzadeh et al [20].To completely understand the user requirement is the important step in the development of mobile cloud computing systems which are described by K.AKamaruddin et al [18],M.Hilty et al [14],V.Florio et al[8], F Lins1[7] Testing is an obvious step that needs to be implemented after a mobile clouding application. Research have shown that a testing can be incremental, which means that after development of every chunk, we have to test the phase. Its importance is reflected in A. Mohsenzadeh et al[20], V. Lelli Dantas[15], D. Kovachev et al [2], L. Murugesan [10], Yonggang Wen et al [6],Performance of a mobile related applications can be improved day by day using different models and techniques that will lead the mobile computing to a much higher level.Many researches have been done for this purpose such as described in N.Fernando et al[1] ,V. Koufi et al[3] ,P. Pouladzadeh et al[4], Y. Wen, Xiaoqing et al[6] , F. Lins1 et al[7],K. Pousttchi et al[9], K.A Kamaruddin et al[18].The great benefit of cloud computing is to transfer a data easily over a wireless medium that gives the attribute of mobility to it and has been discussed by Y. Wen et al [6] ,F. Lins et al [17], D. Sahu et al [19], A. Mohsenzadeh et al[20], C. Shravanthi et al [17].The priority of the paremeters included in table 1 may varies depending upon the scenario in which that application is going to run for example if a device is doing a wireless communication ,mobility parameter will have the higher priority than the device which is just storing the data over the 
data cloud.Flexibility is a factor that can be defined as elasticity in proving better services in order to accommodate users.Surveying different papers, it has been analyzed that some of the papers discusses the issue of flexibility such as in N.Fernando et al[1],D.Kovachev et al[2], Shih-Hao Chang et al[5],Lins1 et al[7], K.Pousttchi et al[9], L.Murugesan et al[10],S.Abolfazli et al[12],A.KBamdra et al[13],V.Lelli Dantaset al[15],K.AKamaruddin[18],A. Mohsenzadeh et al [20]..Researches have been done to reduce flaws in the the previous systems.

Table1. Evalution Cretria for Cloud Computing

\begin{tabular}{|c|c|c|}
\hline Evaluation parameters & Meaning & $\begin{array}{c}\text { Possible } \\
\text { values }\end{array}$ \\
\hline Mobility & Transfer freely anywhere, can run in any environment. & Yes, No \\
\hline Performance & Perform effectively in minimum time. & Yes, No \\
\hline Cost Effectiveness & In minimum cost do better work completely. & Yes, No \\
\hline Privacy & Secure personal information of the user. & Yes, No \\
\hline capability & Have potential to resolve problems related to mobile device. & Yes, No \\
\hline Flexibility & $\begin{array}{c}\text { Elasticity in proving better services in order to accommodate } \\
\text { users. }\end{array}$ & Yes, No \\
\hline Security & Provide safety from the Malicious participator. & Yes, No \\
\hline capacity & Provide storage aptitude to the users. & Yes, No \\
\hline Availability & $\begin{array}{c}\text { Accessible in providing effective services, services that need } \\
\text { continuous information to be available. }\end{array}$ & Yes, No \\
\hline Maintainability & $\begin{array}{c}\text { Preserve the services and information that provided to the } \\
\text { users. }\end{array}$ & Yes, No \\
\hline Functionality & Ability to perform different task to provide better environment & Yes, No \\
\hline
\end{tabular}

\begin{tabular}{|c|c|c|c|c|c|c|c|c|}
\hline S\# & Authors & Maintainability & Capacity & Privacy & Availability & $\begin{array}{c}\text { Cost- } \\
\text { effectiveness }\end{array}$ & Flexibility & Mobility \\
\hline 1 & $\begin{array}{l}\text { N.Fernando et } \\
\text { al, } 2012\end{array}$ & yes & no & no & yes & yes & yes & yes \\
\hline 2 & $\begin{array}{l}\text { D.Kovachev } \\
\text { et al, } 2012\end{array}$ & no & no & no & yes & yes & yes & yes \\
\hline 3 & $\begin{array}{l}\text { V.Koufi } \\
\text { et al, } 2012\end{array}$ & yes & yes & no & yes & yes & no & yes \\
\hline 4 & $\begin{array}{c}\text { P.Pouladzadeh } \\
\text { et al, } 2014\end{array}$ & yes & yes & no & no & yes & no & no \\
\hline 5 & \begin{tabular}{|c|} 
Shih-Hao Chang \\
et al, 2013
\end{tabular} & no & yes & yes & yes & yes & yes & no \\
\hline 6 & $\begin{array}{l}\text { Y.Wen et al, } \\
2014\end{array}$ & yes & yes & no & yes & yes & no & yes \\
\hline 7 & $\begin{array}{c}\text { F. Lins1 et al, } \\
2011\end{array}$ & yes & no & yes & yes & No & yes & yes \\
\hline 8 & $\begin{array}{l}\text { V.Florio et al, } \\
2002\end{array}$ & yes & no & no & yes & yes & no & Yes \\
\hline 9 & $\begin{array}{c}\text { K.Pousttchi et } \\
\text { al, } 2004 \\
\end{array}$ & yes & yes & no & yes & yes & yes & no \\
\hline 10 & \begin{tabular}{|c|}
$\begin{array}{c}\text { L.Murugesan et } \\
\text { al, } 2013\end{array}$ \\
\end{tabular} & no & yes & yes & yes & No & yes & yes \\
\hline 11 & $\begin{array}{l}\text { M. Hamdi et } \\
\text { al,2012 }\end{array}$ & no & no & yes & yes & No & no & yes \\
\hline 12 & $\begin{array}{l}\text { S. Abolfazli et } \\
\text { al, } 2014\end{array}$ & yes & yes & no & no & yes & yes & no \\
\hline 13 & $\begin{array}{c}\text { A.K. Bandara et } \\
\text { al } 2012 .\end{array}$ & yes & no & yes & yes & No & yes & yes \\
\hline 14 & $\begin{array}{l}\text { M. Hilty et al } \\
2006\end{array}$ & yes & no & yes & yes & yes & no & no \\
\hline 15 & $\begin{array}{c}\begin{array}{c}\text { V. Lelli Dantas } \\
\text { et al } 2009\end{array} \\
\end{array}$ & yes & no & yes & yes & No & yes & yes \\
\hline 16 & $\begin{array}{c}\text { H.Q Abdullah et } \\
\text { al } 2012\end{array}$ & no & yes & yes & no & yes & no & yes \\
\hline 17 & $\begin{array}{c}\text { C Shravanthi et } \\
\text { al } 2014\end{array}$ & yes & yes & no & yes & No & no & yes \\
\hline
\end{tabular}


Hira Arshad et al.

\begin{tabular}{|c|c|c|c|c|c|c|c|c|}
\hline \hline 18 & $\begin{array}{c}\text { K.AKamaruddin } \\
\text { et al 2011 }\end{array}$ & no & yes & no & yes & No & yes & no \\
\hline 19 & $\begin{array}{c}\text { D. Sahu et al } \\
2012\end{array}$ & no & yes & yes & yes & No & no & yes \\
\hline 20 & $\begin{array}{c}\text { A. } \\
\text { Mohsenzadeh et } \\
\text { al 2015 }\end{array}$ & no & yes & yes & no & No & yes & no \\
\hline
\end{tabular}

\section{Conclusion}

Cloud computing is the emerging technology for mobile devices .this technology is also known as MCC (Mobile cloud computing). In spite of the benefits and versatility for usage of this technology in multiple applications, there are also involve some security threats because mobile computing systems are more vulnerable to attacks. Research have been done to resolve such issues and it has been concluded that this issue can be resolved using different models but cannot be eliminated $100 \%$.No strategy has been best decided yet to make this technology completely efficient. As this technology works over wireless networks so there is an issue of data processing over the cloud. A CMA model has been introduced after research that will allow the massive data storage and to process the data over cloud without any safety threats

In future, we will make sure to improve our research by using enhanced and modified tools and strategies .Security issues can also be resolved completely but for that we need a plan to use strong strategies so that this issue can be resolved up to $100 \%$.This can be done by exploring this field in its depth and by introducing related technologies that can support mobile cloud computing.

\section{REFERENCES}

[1] Niroshinie Fernando, Seng W. Loke, Wenny Rahayu, "Mobile cloud computing: survey", Australia, 6 June 2012.

[2] Dejan Kovachev, Yiwei Cao and Ralf Klamma , "Mobile Cloud Computing: A Comparison of Application Models", Germany, 2011.

[3] Vassiliki Koufi, Flora Malamateniou, George Vassilacopoulos, Andriana Prentza, "An AndroidEnabled Mobile Framework for Ubiquitous Access to Cloud Emergency Medical Services", Piraeus, Greece, 2012.

[4] Parisa Pouladzadeh, Pallavi Kuhad1, Sri Vijay Bharat Peddi, Abdulsalam Yassine,

[5] Shervin Shirmohammadi, "MOBILE CLOUD BASED FOOD CALORIE MEASUREMENT", Canada, 18 July 2014.

[6] Shih-Hao Chang, Yeong-Sheng Chen, Shin-Ming Cheng, "Detection of Sybil Attacks in Participatory Sensing using Cloud based Trust Management System”, Taiwan, 22 Nov. 2013.

[7] Yonggang Wen, Xiaoqing Zhu, Joel J. P. C. Rodrigues, "Cloud Mobile Media: Reflections and Outlook", VOL. 16, NO. 4, JUNE 2014. F. Lins1,2, R. Medeiros1,2, B. Silva1, A. Souza, B. Stephenson and J. Li "'SSC4Cloud Tooling: An Integrated Environment for the Development of Business Processes with Security Requirements in the Cloud", Brazil,USA, 2011.

[8] Vincenzo De Florio,Geert Deconinck, "On Some Key Requirements of Mobile Application Software", Belgium, 11 Apr 2002.

[9] Key Pousttchi, Martin Schurig, “Assessment of Today's Mobile Banking Applications from the View of Customer Requirements”, 2004.

[10] Logeshwaran Murugesan, Prakash Balasubramanian, "Cloud Based Mobile Application Testing",India,2014.

[11] Mohamed Hamdi,"Security of Cloud Computing, Storage, and Networking”, Tunisia, 25 May 2012.

[12] Saeid Abolfazli, Zohreh Sanaei, Ejaz Ahmed, Abdullah Gani, Rajkumar Buyya, "Cloud-Based Augmentation for Mobile Devices: Motivation, Taxonomies, and Open Challenges", VOL. 16, NO. 1, FIRST QUARTER 2014.

[13] Thein Than Tun, Arosha K. Bandara, Charles Haley, Inah Omoronyia, "Privacy Arguments: Analysing Selective Disclosure Requirements for Mobile Applications",UK, 28 Sept. 2012.

[14] Manuel Hilty, Alexander Pretschner, Christian Schaefer, Thomas Walter,"Usage Control Requirements in Mobile and Ubiquitous Computing Applications", Germany, Oct. 2006. 
[15] Valeria Lelli Leitao Dantas, Fabiana Gomes Marinho, Aline Luiza da Costa, Rossana M. C. Andrade ,"Testing Requirements for Mobile Applications", Brazil, September 2009.

[16] Han Qi . Abdullah Gani ,"Research on Mobile Cloud Computing: Review,Trend and Perspectives", Malaysia, 18 May 2012.

[17] C Shravanthi, H S Guruprasad,"MOBILE CLOUD COMPUTING AS FUTURE FOR MOBILE APPLICATIONS", International Journal of Research in Engineering and Technology, Bangalore, 5 May 2014.

[18] Kamalia Azma Kamaruddin, Nor Shahida Mohamad Yusop, Muhamad Azim Muhamad Ali, "Using Activity Theory in Analyzing Requirements for Mobile Phone Application", Malaysia, 2011.

[19] Deepti Sahu, Shipra Sharma, Vandana Dubey, Alpika Tripathi "Cloud Computing in Mobile Applications", India, Volume 2, Issue 8, August 2012.

[20] A. Mohsenzadeh, "Software Trustworthy Testing Based on Cloud Testing", Iran, 8 January 2015 .

\section{AUTHORS' BIOGRAPHY}

Hira Arshad, Department of computer Science, Student at Fatima Jinnah Women, University The Mall Rawalpindi, Pakistan.

Muqaddas Naz, Department of computer Science, Student at Fatima Jinnah Women, University The Mall Rawalpindi, Pakistan.

Madiha Arshad, Department of Software Engineering, Student at Fatima Jinnah Women, University The Mall Rawalpindi, Pakistan. 